\title{
Sulfadiazine - Chitosan Conjugates and Their Polyelectrolyte Complexes with Hyaluronate Destined to the Management of Burn Wounds
}

\section{Raluca Petronela Dumitriu ${ }^{1}$, Lenuta Profire ${ }^{2, *}$, Loredana Elena Nita ${ }^{1}$, Oana Maria Dragostin ${ }^{2}$, Nicolae Ghetu ${ }^{3}$, Dragoș Pieptu ${ }^{3}$ and Cornelia Vasile ${ }^{1, *}$}

1 "Petru Poni" Institute of Macromolecular Chemistry, Department of Physical Chemistry of Polymers, 41A Grigore Ghica Voda Alley, 700487 Iasi, Romania; E-Mails: rdumi@icmpp.ro (R.P.D.); lnazare@icmpp.ro (L.E.N.)

2 “Grigore T. Popa" University of Medicine and Pharmacy, Faculty of Pharmacy, Department of Pharmaceutical Chemistry, 16 University Street, 700115 Iasi, Romania; E-Mail: oana.dragostin@yahoo.com

3 “Grigore T. Popa" University of Medicine and Pharmacy, Faculty of Medicine, Department of Plastic and Reconstructive Surgery, 16 University Street, 700115 Iasi, Romania; E-Mails: ghetu.nicolae@umfiasi.ro (N.G.); dragos.pieptu@umfiasi.ro (D.P.)

* Authors to whom correspondence should be addressed;

E-Mails: cvasile@icmpp.ro (C.V.); lprofire@umfiasi.ro (L.P.);

Tel.: +40-232-217-454 (L.P.); Fax: +40-232-211-299 (L.P.).

Academic Editor: Heather Sheardown

Received: 24 October 2014 / Accepted: 23 December 2014 / Published: 16 January 2015

\begin{abstract}
In the present study polyelectrolyte complexes (PECs) based on new sulfadiazine-chitosan conjugates with sodium hyaluronate have been developed with potential use in treatment of burn wounds. The PECs were chemically characterized using Fourier Transform-Infrared Spectroscopy, Scanning Electon Microscopy and Near Infrared Chemical Imaging Technique. The swelling behavior and in vitro sulfadiazine release were also investigated. The antimicrobial activity was evaluated towards three bacterial strains: Escherichia coli, Listeria monocytogenes and Salmonella thyphymurium. The developed PECs demonstrated their antimicrobial efficiency against tested bacterial strains, the PECs containing sulfadiazine-modified chitosan being more active than PECs containing unmodified chitosan.
\end{abstract}


Keywords: chitosan; hyaluronic acid; sulfadiazine; polyelectrolyte complex; burn wound

\section{Introduction}

Dressings are applied on open wounds to protect them from injuries and bacteria invasion. An ideal dressing should maintain a moist environment at the wound interface, allow gaseous exchange, act as a barrier to microorganisms, and remove excess exudates. It should also be non-toxic, non-allergenic, non-adherent and be easily removed without trauma; it should be made from a readily available biomaterial that requires minimal processing, possesses antimicrobial properties and promotes wound healing. Dressings made from natural or semisynthetic polymers are increasingly used to deliver drugs to acute, chronic and other types of wounds.

Chitin, chitosan, and their derivatives, prepared in various forms as hydrogels, nanofibers, membranes, micro/nanoparticles and sponges are promising biomaterials for wound dressing and other biomedical applications, such as drug and gene delivery, tissue engineering, etc. [1-5]. These have excellent properties being adhesive, biocompatible, biodegradable, nontoxic, hydrophilic, having also antimicrobial effect and oxygen permeability [6,7].

Chitosan stimulates cell proliferation and histoarchitectural tissue organization has hemostatic activity, which helps in natural blood clotting and blocks nerve endings, reducing pain. Chitosan gradually depolymerizes to release $\mathrm{N}$-acetyl-beta-D-glucosamine, which initiates fibroblast proliferation, helps in ordered collagen deposition and stimulates increased levels of natural hyaluronic acid synthesis at the wound site. It also helps in faster wound healing and scar prevention [8]. Both chitin and chitosan fibers show good mechanical properties as mechanical strength of $1.5-2.5 \mathrm{~g} / \mathrm{dtex}$ and elongation at break $8 \%-20 \%$, being unique as raw materials for hi-tech bandages [9].

Hyaluronic acid (HA) exhibits an enhanced lubricating and water adsorption capacity influencing water retention and cellular events, such as attachment, migration and proliferation [10]. It has been used in ophthalmic surgery, arthritis treatment, in tissue engineering, as component of scaffolds for wound healing and implant devices [11]. The implants covered with HA and its derivatives reduce adsorption, adhesion and cellular proliferation of Staphylococcus aureus at least 100 times [12].

To further improve the chitosan (CS) properties and also to increase the ability as carriers for hydrophilic and lipophylic drugs, the chemical modification and copolymerization are applied, e.g., by reaction with thiazolidinone [13] or by grafting with poly( $\mathrm{N}$-isopropylacrylamide), to obtain temperature-responsive hydrogels [14], by quaternization, grafting with alkyl aldehydes or alkyl ketones, to produce $\mathrm{N}$-alkyl chitosan, with fatty acids, steroid derivatives, poly( $\varepsilon$-caprolactone) as was reviewed by Riva et al. [15-17]. CS-glutamate, CS-succinate, and CS-phthalate provide sustained release in basic medium [18]. There are also studies on chitosan conjugation with different drugs, such as CS-5-fluorouridine conjugate [19] or CS-alendronate conjugate [20]. CS-doxorubicin conjugation was carried out using succinic anhydride as a crosslinker. Trastuzumab was conjugated to self-assembled CS-doxorubicin conjugate (CS-DOX) nanoparticles (particle size, $200 \mathrm{~nm}$ ) via thiolation of lysine residues and subsequent linking of the resulted thiols to chitosan. The monoclonal antibody, trastuzumab, was used as a targeting agent in nanoparticles carrying the antitumor drug, doxorubicin, 
specifically to its site of action [21]. The mucoadhesive property of CS, especially in an acidic $(<\mathrm{pH} 6.0)$ environment, was increased by conjugating an aromatic sulfonamide group at the $\mathrm{C} 2-\mathrm{N}$ position of chitosan. The CS-4-carboxybenzene sulfonamide conjugate showed antibacterial activity against Escherichia coli and Staphylococcus aureus [22,23].

Polyelectrolyte complexes (PECs) can be obtained by electrostatic interaction of amino groups $\mathrm{C}_{2}$ position in glucopyranosic units of $\mathrm{CS}$ with anionic groups (e.g., carboxyl) of polyanions of natural origin (such as pectine, alginate, carrageenan, xanthan gum, carboxymethyl cellulose, chondroitin sulfate, dextran sulfate, hyaluronic acid) or synthetic origin (e.g., polyacrylic acid, polyphosphoric acid, poly(L-lactide) [24]. PECs shows a very important sensibility at swelling, especially by $\mathrm{pH}$ modification, comparatively with covalently crosslinked hydrogels, which lead to the wide variety of applications [25]. PECs protects HA against enzymatic hydrolysis but only at $\mathrm{pH}$ values different of the optimum for enzymatic activity. PEC CS/HA as sponges and films allow the culture of various specific cells as keratocytes, which produces skin matrix accelerating wound healing after skin ablation without inflammatory reactions and toxicity for animals [26]. PEC obtained from chitosan and alginate can be applied to bandages or powders, which protect the wound, accelerates healing and prevent bacterial contamination [27]. Increase of the HA amount weakened the water vapor permeability, bovine albumin adsorption, and fibroblast adhesion, which are desirable characteristics for wound dressing. Comparatively with Vaseline, CS/HA films are more efficient in wound healing because they do not cause damage to the wound when the bandage is removed [28]. A wet treatment can be realized and both components of PEC contribute through their properties to the enhanced antimicrobial activity, and prevent wound damage during treatment. Polyelectrolyte complexation occurs under mild reaction conditions. PEC hydrogels exhibit a highly $\mathrm{pH}$-sensitive swelling, therefore, they can be used for $\mathrm{pH}$-controlled drug delivery in different conditions. The addition of HA to chitosans (chitosan hydrochloride and 5-methyl-pyrrolidinone chitosan) leads to a reduction in wound dressing hydration properties and a modulation of drug release [29].

Sulfonamides and their derivatives are among the most useful antimicrobial agents because of their low cost, low toxicity and excellent activity against bacterial infections [30]. Sulfadiazine (SDZ) is useful in the treatment of meningoccocal, staphyloccocal, streptococcal, etc. infections.

Based on this action, sulfadiazine was selected to modify CS and to obtain formulations with modified and retarded antimicrobial action. The aim of this study is to develop new polyelectrolyte complexes based on a new sulfadiazine-chitosan conjugate (SCS) with hyaluronic acid, as a new way to combine the bacteriostatic effect of chitosan with that of sulfadiazine, to control properties, drug release and also to improve the antimicrobial properties for wound healing application compared with CS/HA PECs in the treatment of the burn wounds.

\section{Results and Discussion}

\subsection{Preparation of SCS/HA PEC Sponges}

PECs are formed based on the ionic interactions established between two oppositely charged polyelectrolytes, thus the $\mathrm{pH}$ value of the solution has to be controlled in order to ensure that both molecules are on the charged form. The suitable $\mathrm{pH}$ of the buffer solution for obtaining CS/HA and 
SCS/HA PECs depends on the pKa of the two polymers, namely the value of 6.5 reported for chitosan [31] and 2.9 for hyaluronate [32]. Selecting a $\mathrm{pH}$ between these two values, the requirements for obtaining PECs should be accomplished (Figure 1).
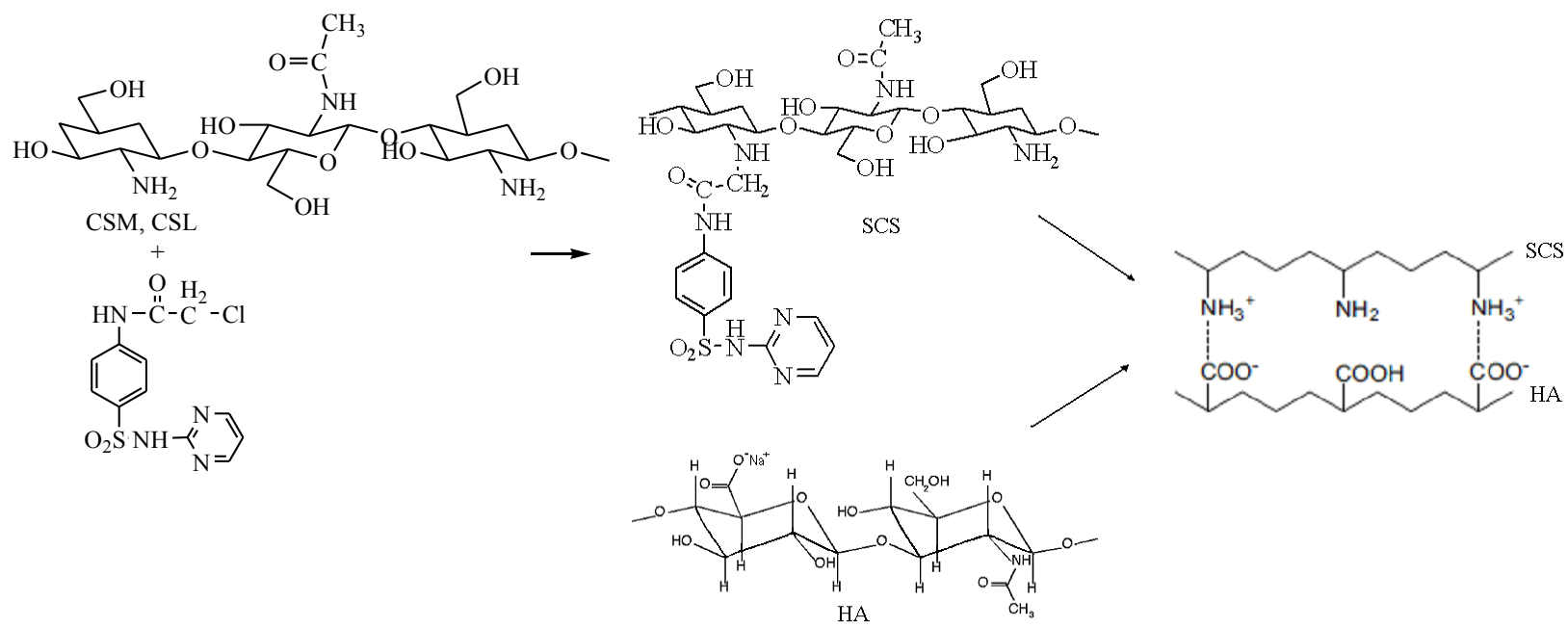

Figure 1. Formation of polyelectrolyte complexes (PECs) with sulfadiazine-chitosan conjugate (SCS) and sodium hyaluronate (HA).

The $\mathrm{pH}$ of 4.12 was chosen for the acetate buffer solution used to obtain the PECs, due to the chitosan poor solubility or low reaction yields obtained at other pHs (3, 3.5, 4.5 and 5). For example, at $\mathrm{pH} \sim 3$, a coacervate was formed as gel spheres but the dry product obtained resulted in a low reaction yield of $\sim 40 \%$ showing that ionic interactions established between the two oppositely charged polyelectrolytes were not sufficient to accomplish PEC formation, because at this $\mathrm{pH}$ value the carboxylic groups of HA are still mostly protonated. Because of the low level of amino groups protonation, the chitosan with medium molecular weight (MMW, DD of $77.56 \%$ ) and SCSM (DS of $31.36 \%$ ) had both a poor solubility at pHs 5 and 4.5 because of a low level of amino groups protonation at this $\mathrm{pH}$. SCS has a poor solubility due to the lower number of amino groups that can be protonated at acidic $\mathrm{pH}$, being substituted with SDZ.

At $\mathrm{pH} 4.12$, the CS and HA solutions were transparent and clear, while SCS (Mm) formed a turbid solution. In case of PECs prepared with chitosan the formation of a white precipitate was observed, while for PECs with sulfadiazine-modified chitosan (SCS) a light yellow precipitate was obtained (Figure 2). The $\mathrm{pH}$ of the solution increased slightly, at 4.62 for PEC M formation and at 4.44 for PEC Mm, which is a proof that various bonds are involved in ionic complex formation. The reaction yields obtained at $\mathrm{pH} 4$ after freeze-drying of the samples are given in Table 1.

The aspect of the dried samples is like xerogels/aerogels for PEC L (chitosan low molecular weight — sodium haluronate) and PEC M (chitosan medium molecular weight—sodium hyaluronate), while PEC Lm (sulfadiazine modified chitosan low molecular weight-sodium hyaluronate) and PEC Mm (sulfadiazine modified chitosan medium molecular weight-sodium hyaluronate) are compact sponges. In the last case, it seems that in the case of sulfadiazine-modified chitosan complexes different kinds of specific interactions as ionic and hydrogen bonding are involved in complex formation therefore the PEC becomes more compact. 


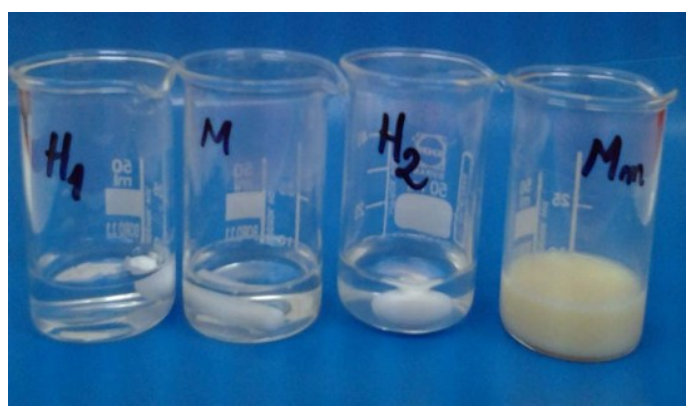

(a)

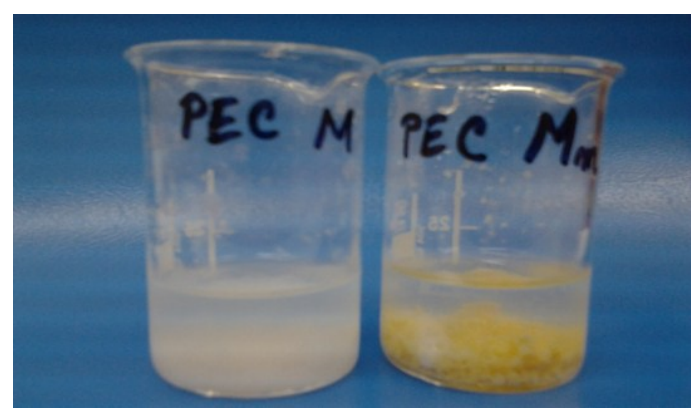

(b)

Figure 2. Images of the chitosan (CS) -M, sulfadiazine modified chitosan (SCS) -Mm and sodium hyaluronate (HA) $-\mathrm{H}_{1}, \mathrm{H}_{2}$ solutions before mixing (a) and after polyelectrolyte complexes (PECs) formation (b).

Table 1. Reaction yields obtained at $\mathrm{pH} 4.12$.

\begin{tabular}{ccc}
\hline PECs samples & Notation & Yield (\%) \\
\hline CSL:HA (1:1) & PEC L & 69.7 \\
CSM:HA (1:1) & PEC M & 65.9 \\
SCSL:HA (1:1) & PEC Lm & 71.6 \\
SCSM:HA (1:1) & PEC Mm & 78.7 \\
\hline
\end{tabular}

CSL: chitosan low molecular weight; CSM: chitosan medium molecular weight; SCSL: sulfadiazine modified chitosan low molecular weight; SCSM: sulfadiazine modified chitosan medium molecular weight; HA: sodium hyaluronate.

\subsection{FT-IR Spectroscopy}

The FT-IR spectra obtained for the PECs prepared and their components are presented in Figure 3a,b, in the spectral regions $2500-4000 \mathrm{~cm}^{-1}$ and $900-1900 \mathrm{~cm}^{-1}$, respectively. In the spectral region $2500-4000 \mathrm{~cm}^{-1}$ there are two absorption bands typically exhibited by polysaccharides: the broad band situated between $3360-3450 \mathrm{~cm}^{-1}$ assigned to the $\mathrm{O}-\mathrm{H}$ stretching vibration $(\mathrm{vOH})$ overlapped with the $\mathrm{N}-\mathrm{H}$ stretching vibrations $(\mathrm{vNH})$ and the band situated around $2921 \mathrm{~cm}^{-1}$ assigned to $\mathrm{C}-\mathrm{H}$ stretching vibrations $(\mathrm{vCH})$.

The broad band assigned to polysacharidic $\mathrm{OH}$ groups from chitosan is shifted to lower wavenumbers in the spectra of both the sulfadiazine-modified CS (SCS) and of PECs showing the presence of interactions between components.

The spectrum of sodium hyaluronate (HA) and of chitosan (CS) exhibit a band at $1626-1658 \mathrm{~cm}^{-1}$ attributed to $\mathrm{C}=\mathrm{O}$ stretching vibrations (amide $\mathrm{I}$ ) from the acetylated units with a shoulder at $1562-1594 \mathrm{~cm}^{-1}$ corresponding to $\mathrm{N}-\mathrm{H}$ bend of amide II overlapped with the $\mathrm{N}-\mathrm{H}$ bending vibration of the amine groups present in the desacetylated units of chitosan.

In the spectra of sulfadiazine-modified chitosan (SCS) and of PECs prepared with SCS (PEC Lm and PEC Mm) the bands corresponding to sulfadiazine (SDZ) can be identified: $\mathrm{C}-\mathrm{H}$ band (para) between $700-895 \mathrm{~cm}^{-1}$ or the more intense bands assigned to $\mathrm{C}-\mathrm{N}$ stretch (aryl) at $1260 \mathrm{~cm}^{-1}$ and to $\mathrm{S}=\mathrm{O}$ stretch at $1082 \mathrm{~cm}^{-1}$ and $1130 \mathrm{~cm}^{-1}$ and some other bands are shifted to lower wavenumbers. In the spectra of PEC Mm the bands assigned to SDZ are more intense than in PEC Lm spectra. 


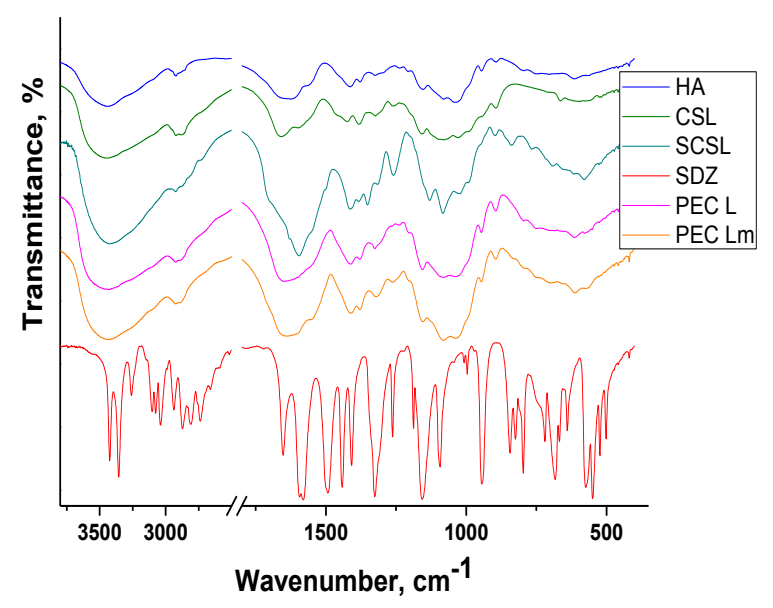

(a)

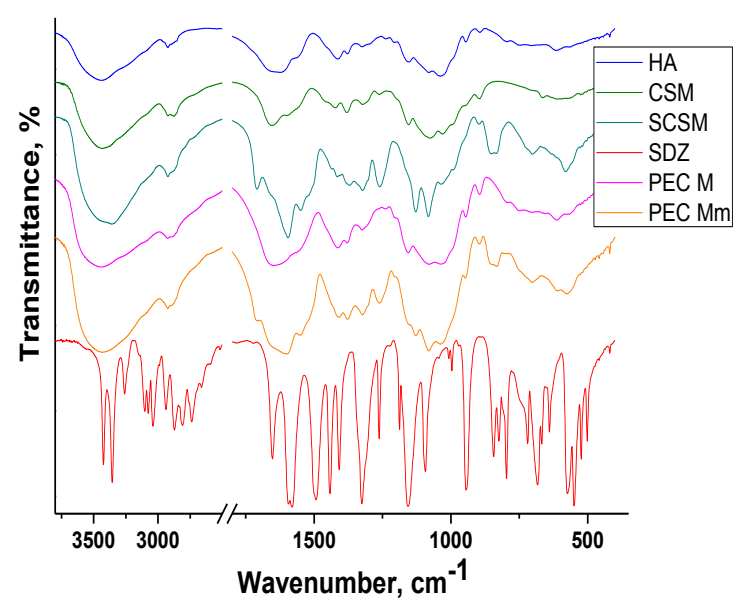

(b)

Figure 3. FT-IR spectra of components and of PEC sponges based on (a) CSL/SCSL and (b) CSM/SCSM (PEG: polyelectrolyte complexes, CSL/SCSL: chitosan low molecular weight/sulfadiazine modified chitosan low molecular weight, CSM/SCSM: chitosan medium molecular weight/sulfadiazine modified chitosan medium molecular weight).

In PECs spectra the bands corresponding to components can be identified, frequently overlapped due to similar structures of the two polysaccharides, chitosan and hyaluronate. In all FT-IR spectra of PECs the bands are wider and slightly shifted compared with the corresponding bands assigned to components, which indicates the presence of interactions leading to PECs formation.

\subsection{Swelling Study}

The swelling measurements performed for PEC samples in PBS of $\mathrm{pH} 7.4$ at $37{ }^{\circ} \mathrm{C}$ (Figure 4) showed the increased and faster swelling degree (SD) of PECs prepared with chitosan and PEC Lm up to $\sim 1700 \%$ compared with PEC Mm, which reaches the maximum SD of $\sim 1400 \%$ at a slower rate. The results obtained suggest that the prepared PEC sponges are quite hydrophilic, having an increased water uptake capacity. High swelling capacity is required for absorption of exudates and also to maintain a moist environment for good wound healing.

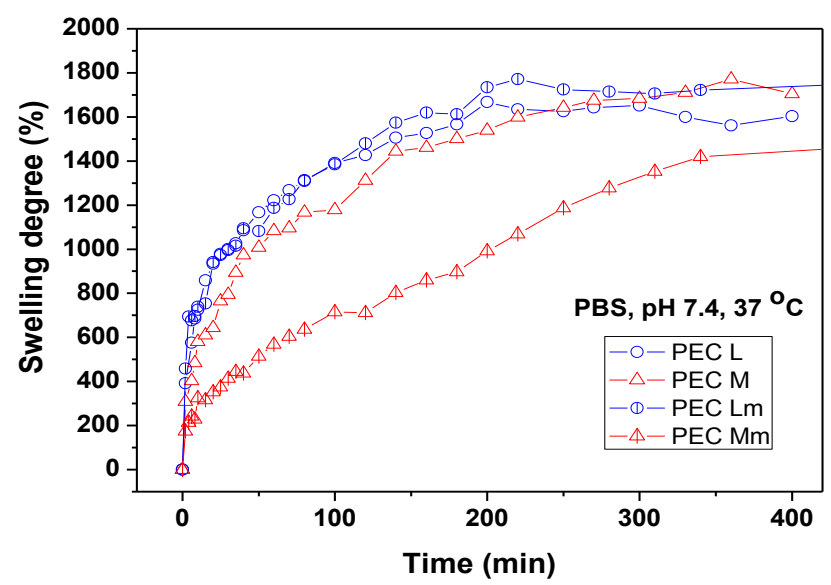

Figure 4. Swelling behavior of PEC (polyelectrolyte complexe) sponges in PBS (phosphate buffered saline) $\mathrm{pH} 7.4$ at $37^{\circ} \mathrm{C}$. 
The swelling properties of the PECs can be explained based on the water-transfer mechanism and kinetics. The swelling kinetic parameters are summarized in Table 2.

Table 2. Swelling kinetic parameters of PEC sponges.

\begin{tabular}{ccccc}
\hline Sample & $\boldsymbol{n}$ & $\mathbf{R}^{\mathbf{2}}$ & $\mathbf{k}\left(\mathbf{m i n}^{\mathbf{n}}\right)$ & $\mathbf{R}^{\mathbf{2}}$ \\
\hline PEC L & 0.33 & 0.999 & $18.76 \times 10^{-2}$ & 0.998 \\
PEC Lm & 0.3 & 0.998 & $20.69 \times 10^{-2}$ & 0.999 \\
PEC M & 0.36 & 0.999 & $13.45 \times 10^{-2}$ & 0.999 \\
PEC Mm & 0.43 & 0.998 & $6.32 \times 10^{-2}$ & 0.998 \\
\hline
\end{tabular}

PEC L (chitosan low molecular weight-sodium haluronate); PEC Lm (sulfadiazine modified chitosan low molecular weight-sodium hyaluronate); PEC M (chitosan medium molecular weight-sodium hyaluronate), PEC Mm (sulfadiazine modified chitosan medium molecular weight-sodium hyaluronate).

The diffusion exponent $(n)$ values suggest that the swelling mechanism of PEC sponges takes place generally by an anomalous transport, which occurs by coupling Fickian diffusion with the relaxation of the hydrogel network. Exception is sample PEC Mm, which has a diffusion coefficient closer to 0.5 $(n=0.43)$, indicating the approach to a Fickian diffusion mechanism. PEC Mm presents the smoothest and slowest swelling profile among the investigated PECs, because of the lowest swelling rate constant value, of $6.32 \times 10^{-2} \mathrm{~min}^{-0.43}$, compared with the other three PECs investigated. This behavior upon swelling correlates with the slower release rate of the drug from this polymeric matrix.

The swelling behavior studies performed at $\mathrm{pH} 7.4$ showed a lower stability of the PECs obtained with SCS compared with PECs prepared with neat CS. Both PEC Lm and PEC Mm maintained compact for weight measurements only up to 300-350 min at $\mathrm{pH}$ 7.4. Taking into consideration the results and observations from the swelling study, the drug release experiment was performed at acidic $\mathrm{pH}$ in order to be able to observe the prolonged SDZ release in time from SCS-based PECs.

\subsection{Scanning Electron Microscopy (SEM)}

The SEM technique allowed observation of the morphologic details of the PEC sponges both on the surface and on the edge/in fracture. In Figure 5 are presented the images obtained, showing a heterogeneous porous morphology with pores highly interconnected.
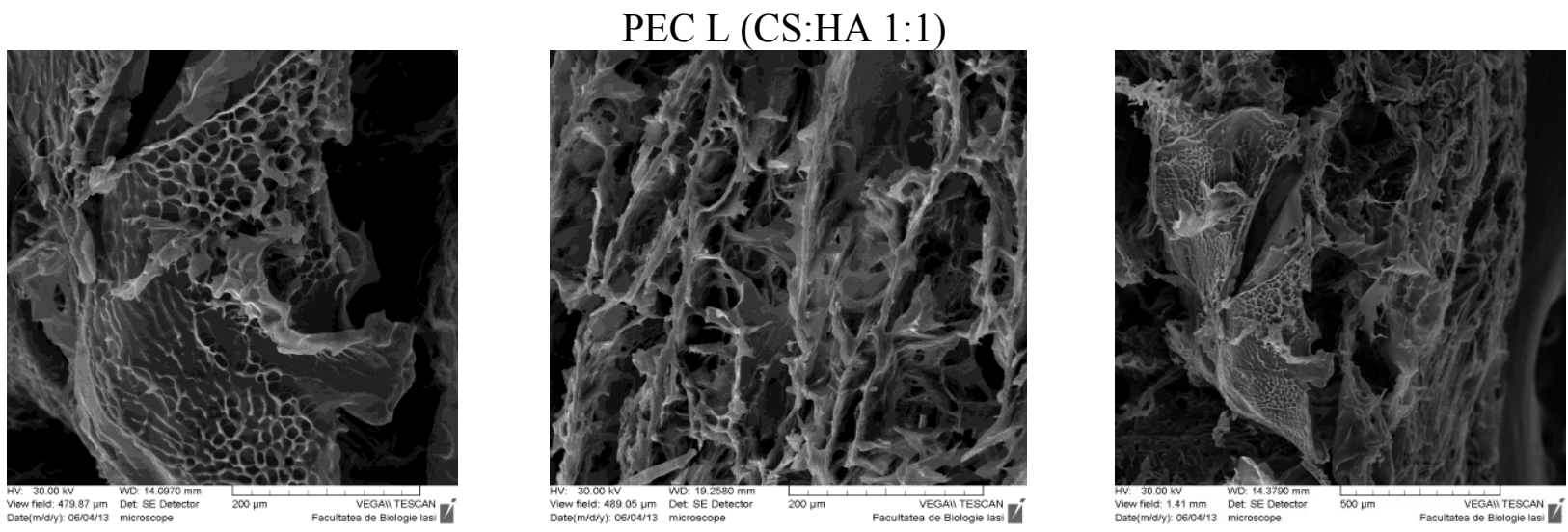

(a)

Figure 5. Cont. 

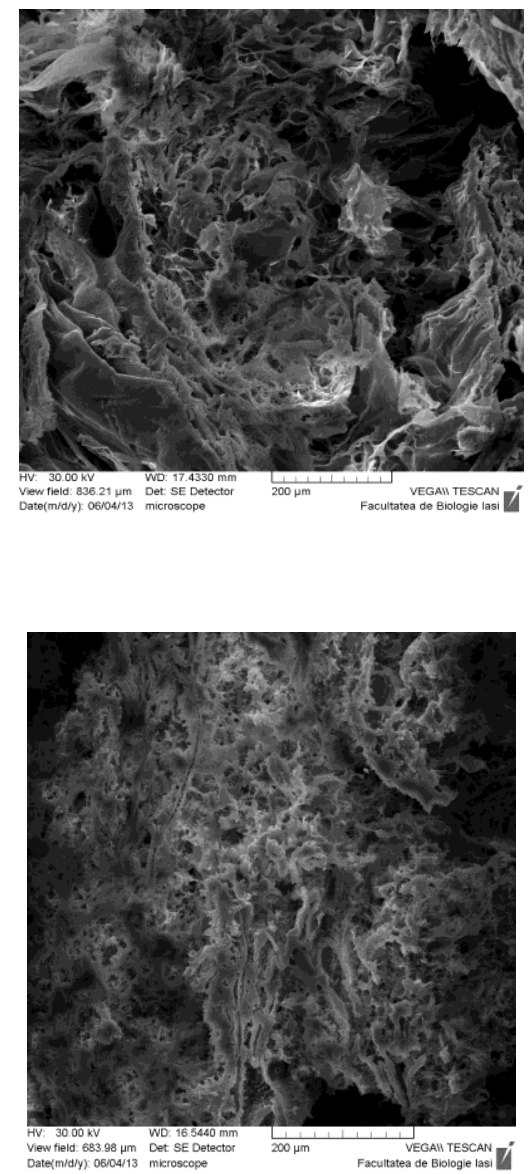

PEC Lm (SCSL:HA 1:1)
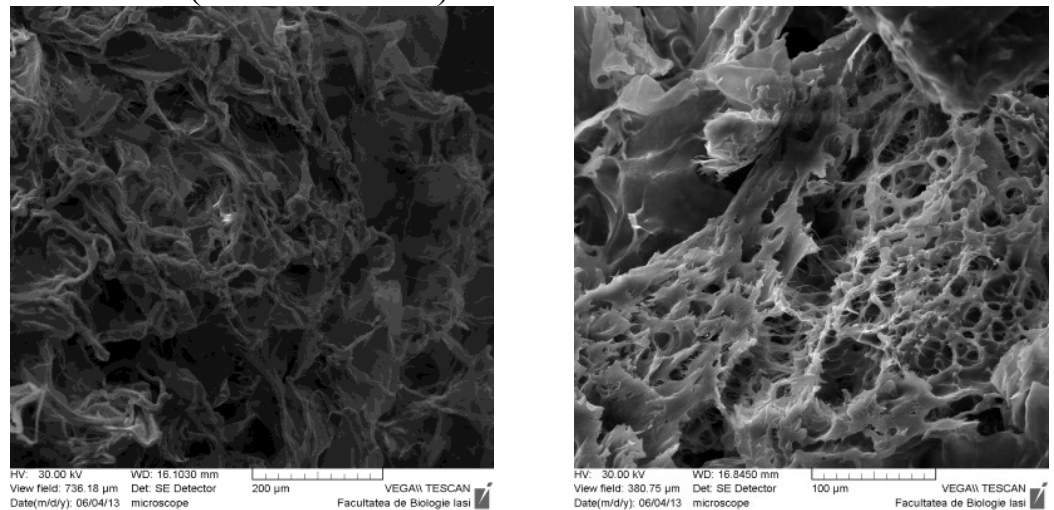

(b)
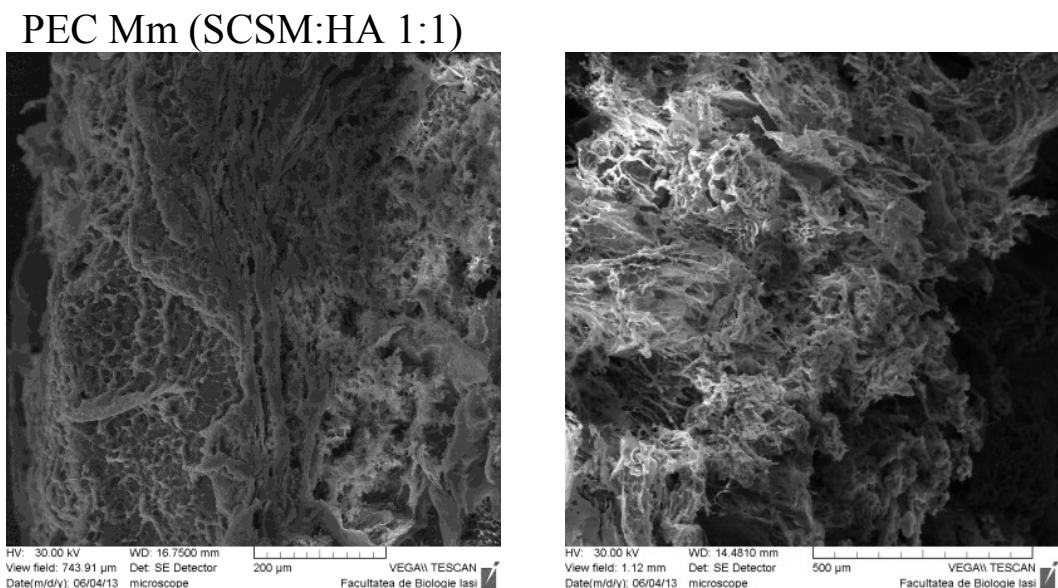

(c)

Figure 5. SEM images of PECs (polyelectrolyte complexes): (a) PEC L (chitosan low molecular weight-sodium hyaluronate); (b) PEC Lm (sulfadiazine modified chitosan low molecular weight-sodium hyaluronate); and (c) PEC Mm (sulfadiazine modified chitosan medium molecular weight-sodium hyaluronate).

The sample PEC Mm seams to have an enhanced and more dense/compact porosity compared with the other samples, which allows an efficient entrapment of the drug within the 3D structure followed by a subsequent slower release.

\subsection{Near Infrared Chemical Imaging (NIR-CI)}

The NIR-CI method of analysis provides information about the spatial distribution of the components with the possibility to determine the degree of the chemical and/or physical heterogenity [33]. The evaluation of the components distribution in samples was made by near infrared chemical imaging (NIR-CI) using chemometric analysis method.

Based on multivariate analysis techniques and principal component analysis (PCA), partial least squares regression (Partial Least Squares-Discriminant Analysis, PLS-DA) and soft independent modeling of class analogy (SIMCA), the quantitative and qualitative information was extracted from the NIR spectral variables data cube. 
The PLS-DA models corresponding to the components (sulfadiazine-SDZ, sulfadiazine-modified chitosan and to PEC samples are depicted in Figure 6. It can be noticed that both the sulfadiazine-modified chitosan samples (SCSL and SCSM) and the PECs obtained (PEC Lm and PEC Mm) have a good homogeneity, but samples SCSM and PEC Mm have a visibly higher homogeneity degree, which corresponds to a more uniform drug distribution within the matrix; data that correlates well with the drug release behavior.

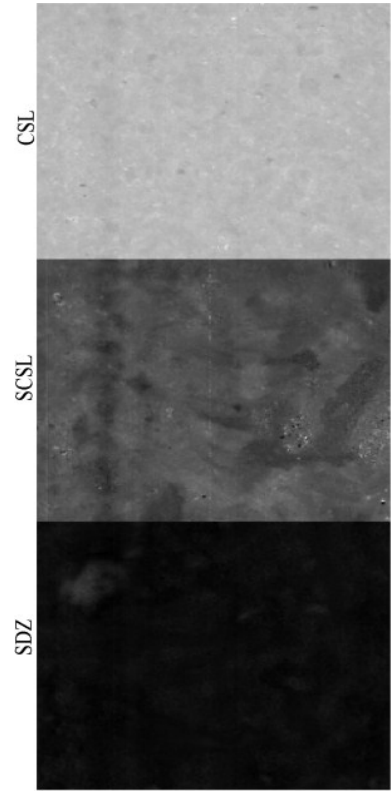

(a)

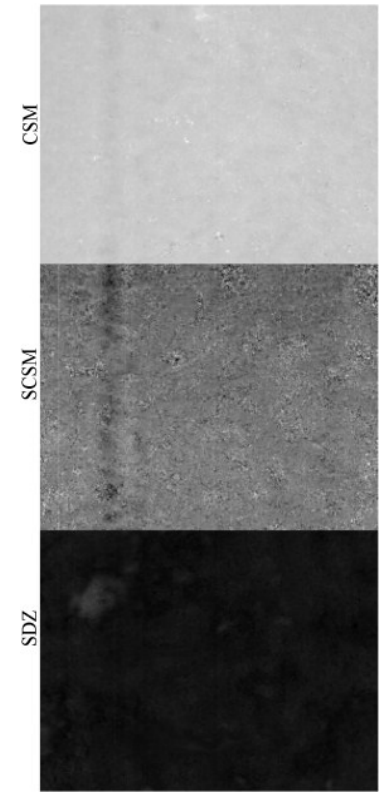

(b)

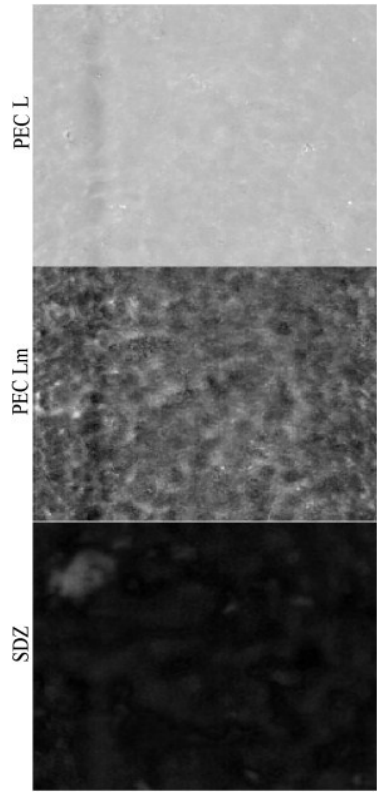

(c)

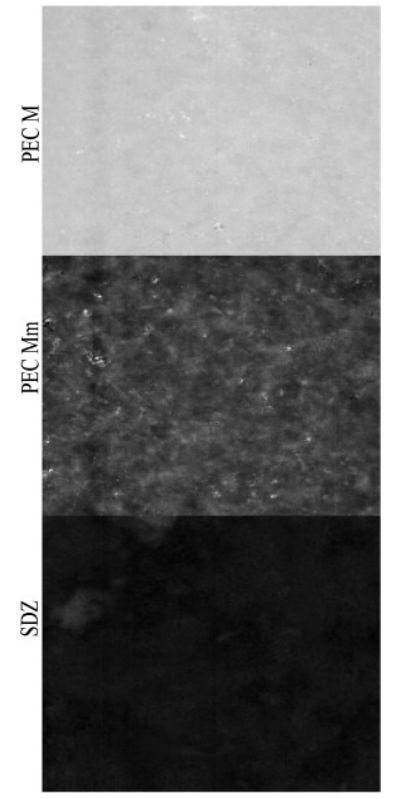

(d)

Figure 6. Partial Least Squares-Discriminant Analysis (PLS-DA) model for sulfadiazine (SDZ)-containing systems: (a) CSL-SCSL-SDZ (chitosan low molecular weight-sulfadiazine modified chitosan low molecular weight-sulfadiazine); (b) CSM-SCSM-SDZ (chitosan medium molecular weight-sulfadiazine modified chitosan medium molecular weight-sulfadiazine); (c) PEC L-PEC Lm-SDZ (chitosan low molecular weight:sodium hyaluronate-sulfadiazine modified chitosan low molecular weight:sodium hyaluronate-sulfadiazine); (d) PEC M-PEC Mm-SDZ (chitosan medium molecular weight:sodium hyaluronate-sulf adiazine modified chitosan medium molecular weight:sodium hyaluronate-sulfadiazine).

The prediction for these samples as non-classified (100\% new compounds both as PEC and SDZ incorporated PECs noted as PEC L-SDZ, PEC Lm-SDZ and PEC M-SDZ, PEC Mm-SDZ, respectively, for low and medium average molecular weight unmodified and modified chitosan) shows that the conjugate with the SDZ was successfully obtained and the PECs were prepared as new and efficient drug release systems.

The Near infrared compared spectra of the PEC hydrogel sponges, sulfadiazine (SDZ) and drug-loaded PECs with an air background are presented in Figure 7. Intense absorption bands corresponding to the functional groups of the components are observed in the full range of the spectra (Table 3). 
Table 3. NIR-CI spectra bands (nm) assignment [34,35].

\begin{tabular}{|c|c|c|c|c|c|}
\hline SDZ & PEC M & PEC M-SDZ & PEC Mm & PEC Mm-SDZ & Bands Assignment \\
\hline $\begin{array}{c}1142,1668, \\
1692 \text { (shoulder) }\end{array}$ & & & & & $\begin{array}{l}\mathrm{C}-\mathrm{H} \text { second overtone; } \\
\mathrm{C}-\mathrm{H} \text { stretch first overtone }\end{array}$ \\
\hline 1475,1525 & 1475 & 1524 & 1490 & 1502 & $\begin{array}{c}\text { NH stretch first overtone, } \\
\text { NH-CO groups } \\
\text { (CO-NHR and } \mathrm{RNH}_{2} \text { groups) }\end{array}$ \\
\hline & $\begin{array}{c}1938 \\
\text { (strong band) }\end{array}$ & & $\begin{array}{c}1938 \\
\text { (strong band) }\end{array}$ & $\begin{array}{c}1940 \\
\text { (wider band) }\end{array}$ & $\begin{array}{l}\mathrm{O}-\mathrm{H} \text { bend second overtone, } \\
\mathrm{C}=\mathrm{O} \text { stretch second overtone }\end{array}$ \\
\hline $\begin{array}{l}1938,1982 \\
2000 \\
\text { (shoulder) }\end{array}$ & & $\begin{array}{c}1938,1982 \\
2000 \\
\text { (shoulder) }\end{array}$ & & & $\begin{array}{c}\mathrm{N}-\mathrm{H} \text { stretch/ } \mathrm{N}-\mathrm{H} \text { in plane bend } \\
\mathrm{C}-\mathrm{N} \text { stretch combination, } \\
\mathrm{NH} \text { bend combination }\end{array}$ \\
\hline & 2032 & & 2036 & $\begin{array}{c}2034 \\
\text { (very weak band) }\end{array}$ & $\mathrm{C}=\mathrm{O}$ stretch second overtone \\
\hline 2057 & & 2059 & & & $\begin{array}{c}\mathrm{N}-\mathrm{H} \text { combinations, } \\
\mathrm{N}-\mathrm{H} \text { in plane bend } \mathrm{C}-\mathrm{N} \text { stretch }\end{array}$ \\
\hline 2096,2150 & 2096 & 2096,2152 & 2098 & 2098 & $\begin{array}{c}\mathrm{C}-\mathrm{H} \text { combination, } \\
\mathrm{R}-\mathrm{NH}_{2} \text { overlapped } \mathrm{O}-\mathrm{H} \text { bend/ } \\
\mathrm{C}-\mathrm{O} \text { stretch combination }\end{array}$ \\
\hline & 2170 & & 2145 & 2155 & $\begin{array}{c}\mathrm{C}-\mathrm{H} \text { deformation combination, } \\
\mathrm{C}=\mathrm{O} \text { stretch combination }\end{array}$ \\
\hline & 2275 & & 2273 & 2273 & $\begin{array}{c}\mathrm{O}-\mathrm{H} \text { stretch/ } \\
\mathrm{C}=\mathrm{O} \text { stretch combination }\end{array}$ \\
\hline & 2326 & & 2326 & 2328 & C-H stretch \\
\hline
\end{tabular}

SDZ-sulfadiazine; PEC M-chitosan medium molecular weight:sodium hyaluronate; PEC M-SDZ —chitosan medium molecular weight: sodium hyaluronate: sulfadiazine; PEC Mn—sulfadiazine modified chitosan medium molecular weight:sodium hyaluronate; PEC Mn-SDZ—sulfadiazine modified chitosan medium molecular weight:sodium hyaluronate: sulfadiazine.

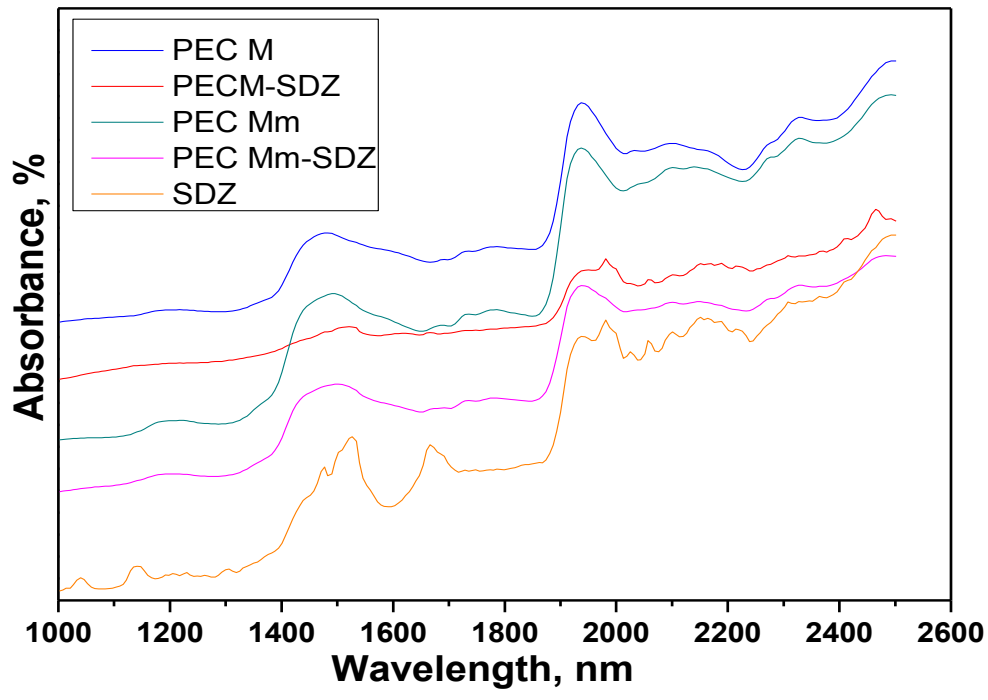

Figure 7. Compared Near-IR reflectance spectra of the polyelectrolyte complexe (PEC) sponges prepared without added sulfadiazine (SDZ) and of SDZ-loaded PECs (PEC M and PEC Mm). 
Sulfadiazine (SDZ) near-IR spectra evidenced the bands at $1142 \mathrm{~nm}$ corresponding to $\mathrm{C}-\mathrm{H}$ second overtone and the band at $1668 \mathrm{~nm}$ with a shoulder at $1692 \mathrm{~nm}$ corresponding to $\mathrm{C}-\mathrm{H}$ stretch first overtone, which can be attributed to $\mathrm{ArCH}$ from benzene ring and pyrimidine ring, respectively. Specific for SDZ are the bands at $1475 \mathrm{~nm}$ and $1525 \mathrm{~nm}$ attributed to $\mathrm{N}-\mathrm{H}$ stretch first overtone (CONHR and $\mathrm{RNH}_{2}$ groups) and the weak band at $1938 \mathrm{~nm}$ coupled with the sharp band at $1982 \mathrm{~nm}$ with a shoulder at $2000 \mathrm{~nm}$ for $\mathrm{N}-\mathrm{H}$ stretch/ $\mathrm{N}-\mathrm{H}$ in plane bend $\mathrm{C}-\mathrm{N}$ stretch combination corresponding to $\mathrm{NH}_{2}$ group and $-\mathrm{NH}$ bonded to pyrimidine ring.

In the spectra of PEC M (CHTM: HA 1:1), a broad band appears at $1475 \mathrm{~nm}$ corresponding to $\mathrm{NH}$ stretch first overtone assigned to amino/ $\mathrm{NH}_{2}$ groups of chitosan and amide/ $\mathrm{NH}-\mathrm{CO}$ groups from both chitosan and hyaluronate and an intense/strong band at $1938 \mathrm{~nm}$ corresponding probably to both $\mathrm{O}-\mathrm{H}$ bend second overtone (polysaccharidic $\mathrm{OH}$ groups) and to $\mathrm{C}=\mathrm{O}$ stretch second overtone (from both $\mathrm{COO}^{-}$or amide $\mathrm{NH}-\mathrm{CO}$ groups).

The spectra recorded for PEC Mm and PEC Mm-SDZ are quite similar with that of PEC M, but the broad band recorded at $1475 \mathrm{~nm}$ for PEC M is shifted to $1490 \mathrm{~nm}$ for PEC Mm and to $1502 \mathrm{~nm}$ for PEC Mm-SDZ and changes slightly the aspect for the last one showing the influence of the CONHR and $\mathrm{RNH}_{2}$ groups from SDZ with the peaks recorded at $1475 \mathrm{~nm}$ and $1525 \mathrm{~nm}$. For PEC M-SDZ this band is shifted to $1524 \mathrm{~nm}$ and has a significantly modified aspect and decreased intensity.

The intense/strong band attributed to polysaccharidic OH groups at $1938 \mathrm{~nm}$ in PEC M spectra is shifted to $1940 \mathrm{~nm}$ and becomes larger and less intense in the spectra of PEC Mm-SDZ, being overlapped with the intense bands from SDZ corresponding to $\mathrm{NH}$ combinations. These particular bands and the ones recorded at $2057 \mathrm{~nm}$ and to $2096 \mathrm{~nm}, 2150 \mathrm{~nm}$ (corresponding to $\mathrm{N}-\mathrm{H}$ in plane bend $\mathrm{C}-\mathrm{N}$ stretch and to $\mathrm{C}-\mathrm{H}$ combination) can be easily identified in the spectra of PEC M-SDZ at almost the same wavelengths as in SDZ spectra.

Meanwhile the spectra of PEC M, PEC Mm and PEC Mm-SDZ present larger bands, with lower intensity in the range $2000-2040 \mathrm{~nm}$. The band at $2032 \mathrm{~nm}$ from PEC M spectra attributed to $\mathrm{C}=\mathrm{O}$ stretch second overtone is slightly shifted to $2036 \mathrm{~nm}$ in the PEC Mm spectra and almost cannot be identified in the spectra of PEC Mm-SDZ which suggests the interactions between $\mathrm{COO}^{-}$groups from $\mathrm{HA}$ with $\mathrm{NH}_{2}$ groups of SDZ. The bands at $2096 \mathrm{~nm}$ and $2170 \mathrm{~nm}$ from PEC M spectra are slightly shifted in PEC Mm spectra, but became larger, with lower intensity and shifted at $2098 \mathrm{~nm}$ and $2155 \mathrm{~nm}$, respectively, in PEC Mm-SDZ spectra, demonstrating the presence of additional SDZ in sample.

These findings show that SDZ bands can be identified in the spectra of the physically loaded PEC sample, while the spectra of the PEC prepared with SDZ-modified CS (PEC Mm) was quite similar with that of PEC M, with larger bands such as PECs present generally, demonstrating that SDZ was well-bonded in the SDZ-CS conjugate as the NIR predictions identified the PEC obtained as a new compound.

The presence of mainly broad bands in the spectra of the PECs obtained suggests the presence of the ionic interactions between the two oppositely charged polyelectrolytes, chitosan and hyaluronate, leading to the formation of PECs. 


\subsection{Drug Release Kinetic Studies}

The in vitro sulfadiazine release was investigated at $37{ }^{\circ} \mathrm{C}$, in acidic solution of $\mathrm{pH}$ 2.7. The drug release profiles are depicted in Figure 8. The sulfadiazine release kinetic parameters were calculated (Table 4).

The acidic $\mathrm{pH}$ of 2.7 was chosen for the drug release study in order to be able to observe the prolonged SDZ release from PECs based on SCS, which demonstrated a reduced stability at pH 7.4 compared with CS-based PECs during swelling studies.

The release rate and the amount of sulfadiazine released depends on the type of sample, either it is a conjugate or a PEC. Also the stability of the PECs in the dissolution medium is affected by their composition.

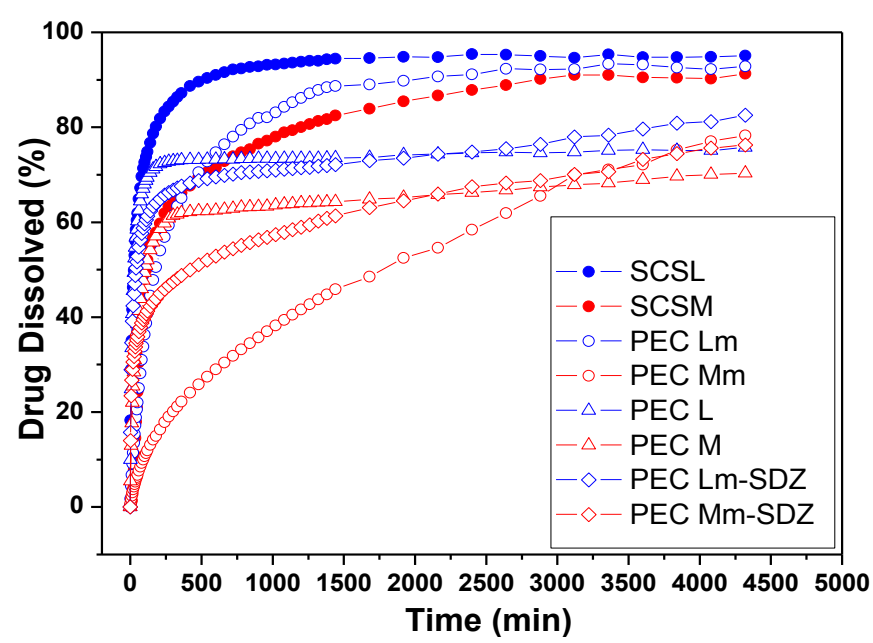

Figure 8. Sulfadiazine release profiles from chitosan (CS) conjugates (SCSL_-sulfadiazine modified chitosan low molecular weight and SCSM-sulfadiazine modified chitosan medium molecular weight) and from SDZ-loaded PECs.

Table 4. Kinetic parameters for sulfadiazine (SDZ) release.

\begin{tabular}{ccccc}
\hline Sample & $\boldsymbol{n}$ & $\mathbf{R}$ & $\mathbf{k} \cdot\left(\mathbf{m i n}^{\mathbf{n}}\right)$ & $\mathbf{R}$ \\
\hline PEC L-SDZ & 0.36 & 0.997 & $3.58 \times 10^{-2}$ & 0.999 \\
PEC Lm & 0.32 & 0.997 & $1.60 \times 10^{-2}$ & 0.999 \\
PEC Lm-SDZ & 0.24 & 0.999 & $4.34 \times 10^{-2}$ & 0.999 \\
SCSL & 0.30 & 0.999 & $3.53 \times 10^{-2}$ & 0.999 \\
PEC M-SDZ & 0.33 & 0.999 & $2.55 \times 10^{-2}$ & 0.999 \\
PEC Mm & 0.23 & 0.999 & $7.45 \times 10^{-3}$ & 0.999 \\
PEC Mm-SDZ & 0.18 & 0.999 & $3.83 \times 10^{-2}$ & 0.999 \\
SCSM & 1.00 & 0.991 & $8.94 \times 10^{-4}$ & 0.998 \\
\hline
\end{tabular}

PEC L-SDZ: chitosan low molecular weight:sodium hyaluronate:sulfadiazine; PEC Lm: sulfadiazine modified chitosan low molecular weight-sodium hyaluronate; PEC Lm-SDZ: sulfadiazine modified chitosan low molecular weight-sodium hyaluronate: sulfadiazine; SCSL: sulfadiazine modified chitosan low molecular weight, PEC M-SDZ: chitosan medium molecular weight:sodium hyaluronate: sulfadiazine; PEC Mm: sulfadiazine modified chitosan medium molecular weight-sodium hyaluronate; PEC Mm-SDZ: sulfadiazine modified chitosan medium molecular weight-sodium hyaluronate: sulfadiazine; SCSM: sulfadiazine modified chitosan medium molecular weight. 
Previous studies performed on chitosan polyelectrolyte complexes and in particular PECs with hyaluronate have shown that complex stability is closely related with the chitosan DA (degree of acetylation), by decreasing when DA increases, the distance between charged groups $\left(\mathrm{NH}_{3}{ }^{+}\right)$increases . Thus the complex is less cooperative for large DA [36].

In our investigations the PECs obtained with SCS were more stable at acidic $\mathrm{pH}$ compared with the PECs obtained with neat CS, which disintegrate at $\mathrm{pH} 2.7$ due to chitosan solubility by protonation of amino groups. SCSM has a DD (degree of desacetylation) of $77.56 \%$ (DA of $22.44 \%$ ) so there was enough charge density at acidic $\mathrm{pH}$ to form a PEC with HA, but due to the increased DS of $31.36 \%$ a significantly lower number of $\mathrm{NH}_{2}$ groups were available for protonation in acidic media to induce solubility compared with CS or SCSL (DS of 16.66\%). Thus by substitution with SDZ the PECs prepared with SCS became more stable at $\mathrm{pH} 2.7$.

The SDZ release results showed a slower release rate from all samples containing CSM, either SCSM or PEC Mm, compared with samples containing CSL/SCSL. Also the release of up to $80 \%$ of SDZ in $72 \mathrm{~h}$ was recorded from almost all PEC samples, except PEC Lm, which has a similar behavior with SCSL. These findings demonstrate that the drug was well entrapped in the polymer matrix, facilitating its slower release.

For the PEC samples prepared with SCSM the release profiles are visibly smoother compared with the other samples, showing the slowest release rate and the lowest release percent during $50 \mathrm{~h} / \sim 2$ days. In particular the PEC Mm samples had a significantly slower release rate compared with PEC M and SCSM.

The slowest release rate was recoded for PEC Mm sample. The enhanced chains length/entanglement in CSM is favorable for keeping the drug inside the polymer matrix for longer time and releasing it slowly subsequently. Additionally, the presence of interchain hydrogen bonding might be also responsible for a better entrapment of the drug within the polymeric matrix and its slower release.

The values obtained for the diffusion exponent $(n)$ at $\mathrm{pH} 2.7$ correspond to an anomalous, non-Fickian mechanism for all samples, except sample SCSM $(n=1)$ when a case II transport mechanism is involved with zero order kinetics.

The decrease of the release rate constant values $(\mathrm{k})$ for all SCSM-containing samples corresponds to a slower release rate and indicates a significantly prolonged delivery from samples containing CSM and especially SCSM.

The release studies were performed at $\mathrm{pH} 2.7$ were all PECs show the approximately the same resistance and aspect. PECs of both modified chitosans are stable over a wide range of $\mathrm{pH}$ from $\mathrm{pH} 2.7$ to 7.4 as also other authors reported [37] while PECs containing SDZ chitosan although they disintegrate at $\mathrm{pH} 7.4$ still remain non-soluble. Therefore both types of PECs matrices could be useful for wound dressing where $\mathrm{pH}$ is higher than 4 .

\subsection{Antimicrobial Tests}

The antimicrobial activity of the PECs obtained with CS, with SDZ-modified CS and with added SDZ to the PECs prepared was studied comparatively on three bacterial strains: Escherichia coli, Listeria monocytogenes and Salmonella thyphymurium. The results obtained are presented in Table 5. 
Table 5. Antimicrobial tests results.

\begin{tabular}{|c|c|c|c|c|c|c|}
\hline \multirow{3}{*}{ Sample } & \multicolumn{6}{|c|}{ Inhibition (\%) } \\
\hline & \multicolumn{2}{|c|}{ Escherichia coli } & \multicolumn{2}{|c|}{ Listeria monocytogenes } & \multicolumn{2}{|c|}{ Salmonella thyphymurium } \\
\hline & $24 \mathrm{~h}$ & $48 \mathrm{~h}$ & $24 \mathrm{~h}$ & $48 \mathrm{~h}$ & $24 \mathrm{~h}$ & $48 \mathrm{~h}$ \\
\hline PEC L & 29 & 38 & 58 & 89 & 17 & 43 \\
\hline PEC M & 49 & 67 & 66 & 83 & 39 & 52 \\
\hline PEC Lm & 59 & 63 & 77 & 100 & 100 & 100 \\
\hline PEC Mm & 52 & 79 & 64 & 92 & 31 & 81 \\
\hline PEC L-SDZ & 49 & 71 & 45 & 77 & 25 & 50 \\
\hline PEC M-SDZ & 68 & 90 & 74 & 100 & 87 & 100 \\
\hline PEC Lm-SDZ & 83 & 95 & 100 & 100 & 94 & 100 \\
\hline PEC Mm-SDZ & 72 & 94 & 70 & 94 & 96 & 88 \\
\hline
\end{tabular}

PEC L: chitosan low molecular weight:sodium haluronate, PEC M: chitosan medium molecular weight:sodium haluronate, PEC Lm: sulfadiazine modified chitosan low molecular weight-sodium hyaluronate, PEC Mm: sulfadiazine modified chitosan medium molecular weight-sodium hyaluronate, PEC L-SDZ: chitosan low molecular weight:sodium haluronate:sulfadiazine, PEC M-SDZ: chitosan medium molecular weight:sodium haluronate:sulfadiazine, PEC Lm-SDZ: sulfadiazine modified chitosan medium molecular weight-sodium hyaluronate-sulfadiazine, PEC Mm-SDZ: sulfadiazine modified chitosan medium molecular weight-sodium hyaluronate: sulfadiazine.

The results obtained confirm the antimicrobial effect of CS-containing PECs ranging from $17 \%$ to $67 \%$ while SDZ has an inhibitory capacity of $70 \%-78 \%$, while the SDZ/CS-containing samples have a significantly enhanced efficiency in activity, reaching even 100\% inhibition of bacteria growth - mostly for samples with added SDZ proving their synergistic effect. For all three bacterial strains investigated, PECs Lm and Mm have an increased inhibition effect compared with CS-based PECs (PEC L and PEC M), while for the PECs with added SDZ, in particular PEC Lm-SDZ and PEC Mm-SDZ, the inhibition percent is even higher. Also the increase of antimicrobial activity was observed for PEC L and PEC M when SDZ was added (Figure 9). No differences are found in antimicrobial activity of the PECs containing CS with low and medium average molecular weight.

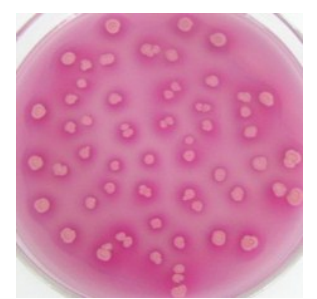

PEC L

PEC M

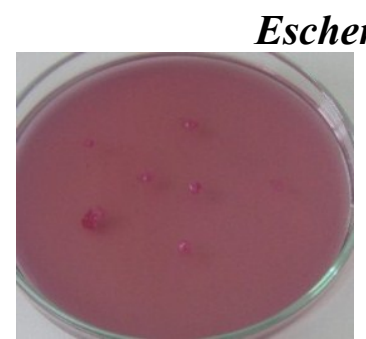

PEC L-SDZ

PEC M-SDZ

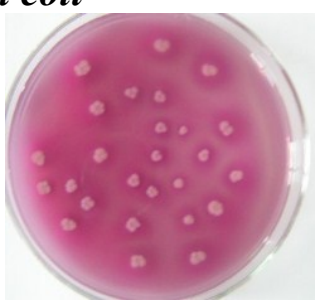

PEC Lm

PEC Mm

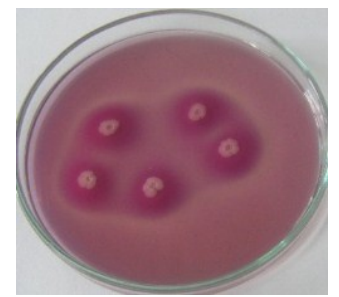

PEC Lm-SDZ

PEC Mm-SDZ

Figure 9. Cont. 


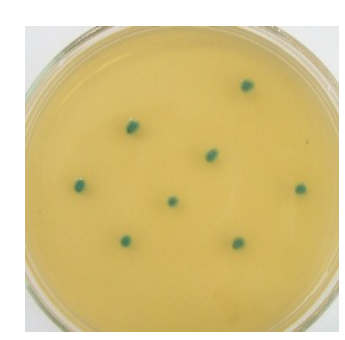

PEC L

PEC M

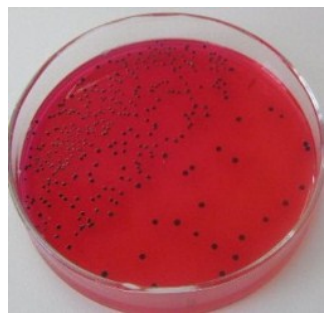

PEC L

PEC M

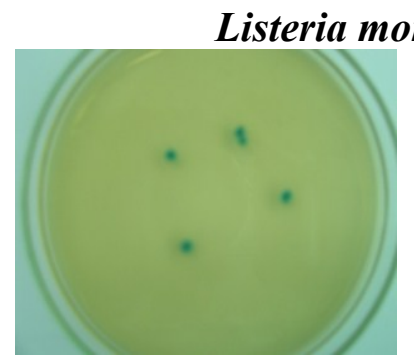

PEC L-SDZ

PEC M-SDZ

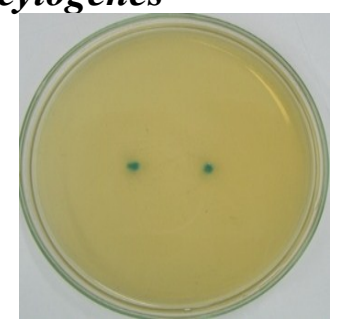

PEC Lm

PEC Mm

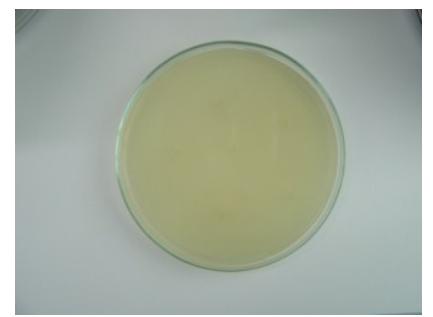

PEC Lm-SDZ

PEC Mm-SDZ

\section{Salmonella thyphymurium}

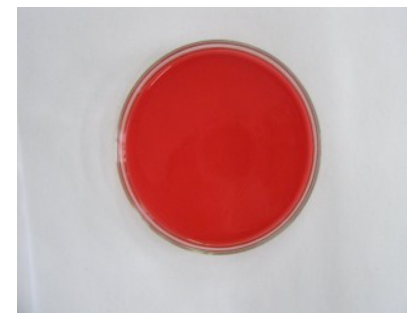

PEC L-SDZ

PEC M-SDZ

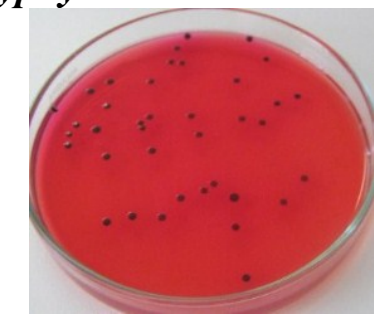

PEC Lm

PEC Mm

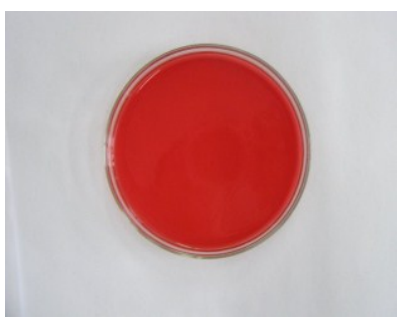

PEC Lm-SDZ

PEC Mm-SDZ

Figure 9. Representative microscopical aspects of the colonies of Escherichia coli, Listeria monocytogenes and Salmonella thyphymurium grown over polyelectrolyte complexes based chitosan/sodium hyaluronate (CS/HA PECs) without and with sulfadiazine (SDZ) modification.

\section{Experimental Section}

\subsection{Materials}

Low (CSL) and medium (CSM) molecular weight chitosan were purchased from Sigma-Aldrich and sodium hyaluronate (HA) from Streptococcus equis was obtained from Fluka. Chitosan (CSL), a product of low molecular weight has a viscosity of a $5 \mathrm{wt} \%$ concentration solution of $20.000 \mathrm{cP}$, in $1 \%$ acetic acid $\left(25^{\circ} \mathrm{C}\right)$ and deacetylation degree of $77.8 \%$. Chitosan (CSM), a product of medium molecular weight $(\mathrm{Mw})$ of 190-310 kDa was purchased from Sigma-Aldrich. It has a viscosity of $200.000 \mathrm{cP}$, in $1 \%$ acetic acid $\left(25^{\circ} \mathrm{C}\right)$ and deacetylation degree of $78.5 \%$.

Sulfadiazine (SDZ), a sulfonamide drug, was used for chitosan modification. The sulfonamides are synthetic bacteriostatic antibiotics with a wide spectrum against most gram-positive and many gram-negative microorganisms. Sulfadiazine is one of the short-acting sulfonamides used for the treatment of rheumatic fever and meningococcal meningitis. Sulfadiazine can be easily identified, being readily absorbed after oral administration and subsequently excreted largely in the urine [38]. As silver salt sulfadiazine is used locally to prevent and treat wound infections in patients with serious burns [39].

Sulfadiazine, also called sulfapyrimidine or 4-amino-N-(pyrimidin-2-yl)-benzen-sulfonamide is a white or white-yellow powder, sensitive to light with a melting point of $252-256{ }^{\circ} \mathrm{C}$, insoluble in water, alcohol, ether, chloroform, soluble in acetone and diluted acidic or alkaline solutions [40]. 
By using sulfadiazine it was expected to increase the antimicrobial effect of the PECs obtained using SDZ-modified CS (SCS). The procedure of synthesis of the CS derivative with sulfadiazine is described in other paper [41].

\subsection{Preparation of SDZ-Modified CS}

The sulfadiazine-modified chitosan derivatives have been synthesized by reaction of chitosan medium molecular weight (CSM) and chitosan low molecular weight (CSL) with N-chloracethyl sulfadiazine using similar methods from the literature applied to other chitosan derivatives [42-44]. To a stirred solution of chitosan (CSM, CSL) $(0.011 \mathrm{M})$ in $1 \%$ acetic acid $(100 \mathrm{~mL})$ a solution of N-chloracethyl sulfadiazine $(0.0132 \mathrm{~mol})$ in DMFA $(50 \mathrm{~mL})$ was added. The reaction mixture was stirred for about $24 \mathrm{~h}$ at room temperature and then the $\mathrm{pH}$ was corrected at 9 with $15 \% \mathrm{NaOH}$ solution when the solid product precipitated. The products were washed five times with water, until the $\mathrm{pH}$ of the filtrate was 7. The final compounds were purified by dialysis against deionized water for 5 days and then freeze-dried on Alpha 1-2 LD Plus lyophiliser (Germany). The degree of substitution of this derivative was $31.64 \%$. Both ionic and covalent bonds could be formed between CS and SDZ as can be remarked from the Figure 1.

\subsection{Preparation of SCS/HA PEC Sponges}

The SCS/HA and CS/HA PECs have been prepared by mixing the two polysaccharide solutions in 1:1 mass ratio, followed by freeze-drying of the insoluble polyelectrolyte complexes formed. Therefore $1 \mathrm{wt} \%$ solutions of HA and CS, respectively, SCS were prepared separately by dissolving $0.1 \mathrm{~g}$ of the polymer in $10 \mathrm{~mL}$ of a $0.1 \mathrm{M}$ acetate buffer $(\mathrm{pH}=4.12)$ solution. The two solutions were then mixed at room temperature in order to prepare the PECs: the CS/SCS solution was kept under continuous stirring $(700 \mathrm{rpm})$ while the HA solution was dropped slowly into the chitosan solution and the mixture was left under vigorous stirring $(1000 \mathrm{rpm})$ for $30 \mathrm{~min}$. The formed PECs were isolated by centrifugation at $5000 \mathrm{rpm}$ for $5 \mathrm{~min}$, washed repeatedly with twice distilled water then were frozen and finally freeze dried overnight. UV-VIS spectra certified the purity of the systems.

\subsection{Investigation Methods}

\subsubsection{FT-IR Spectroscopy}

Fourier Transform-Infrared (FT-IR) spectroscopy analysis was performed on HA, CS, SCS, SDZ and PECs in the range of 4000-500 $\mathrm{cm}^{-1}$, using a Bruker VERTEX 70 spectrometer (Billerica, MA, USA) in Transmittance mode. The dried formulations were grounded to powder, mixed with $\mathrm{KBr}$, compressed into a tablet and then spectra were recorded at $4 \mathrm{~cm}^{-1}$ resolution.

\subsubsection{Swelling Behavior}

The swelling behavior was studied by immersing dried pre-weight pieces of the prepared PEC

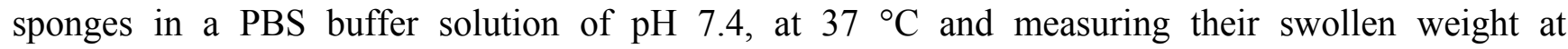
predetermined time intervals. The swelling degree (SD) was evaluated using Equation (1) [45]: 


$$
S D(\%)=\frac{\left(m-m_{0}\right)}{m_{0}} \times 100
$$

where $m_{0}$ is the mass of dry sample and $m$ is the mass at moment " $\mathrm{t}$ " of swelling.

\subsubsection{Scanning Electron Microscopy (SEM)}

The morphology of SCS/HA PEC sponges was analyzed by SEM. Samples were placed on a double-sided graphite tape, attached onto a metal support and coated with gold on a sputtering coater. Observations were performed with a VEGA II TESCAN scanning electron microscope. Magnification is indicated on pictures.

\subsubsection{Near Infrared Chemical Imaging (NIR-CI) Technique}

NIR spectra were recorded on a SPECIM'S Ltd. Sisu-CHEMA, Finland controlled with Evince software package for processing the original image data. The system includes a Chemical Imaging Workstation for 1000 to $2500 \mathrm{~nm}$ NIR domains. The original image for each sample was taken with a NIR model spectral camera, respectively, an imaging spectrograph type ImSpector N17E with 320 and 640 pixel spatial resolution at a rate of 60 to $350 \mathrm{~Hz}$. Each sample was scanned between 1000 and $2500 \mathrm{~nm}$.

The NIR-CI data were collected on a SisuCHEMA (Finland) device, which employs SPECIM's hyperspectral imaging technology on full NIR (1000-2500 $\mathrm{nm}$ ) range. The system is equipped with a spectral camera, $320 \times 640$ pixel spatial resolution for a rate of $60-350 \mathrm{~Hz}$. The hyperspectral camera has a mercury cadmium telluride (MCT) detector. The EVINCE image processing software was used for data analysis.

\subsubsection{In Vitro Drug Release Study}

The in vitro release studies for sulfadiazine (SDZ) have been performed using a 708-DS Dissolution Aparatus coupled with a Cary 60 UV-VIS spectrophotometer (Agilent Technologies). The experiments were carried out in a medium, which mimic the gastrointestinal environment, using an acidic solution of $\mathrm{pH} 2.7$ as dissolution medium. During the experiment the temperature was maintained at $37{ }^{\circ} \mathrm{C}$. Aliquots of the medium of $10 \mathrm{~mL}$ withdrawn at predetermined time intervals were analyzed at $\lambda_{\max }$ of $260 \mathrm{~nm}$, the characteristic wavelength for SDZ. The drug release kinetics was evaluated with a semi-empirical Equation (2) based on Korsmeyer-Peppas model, which is applied at the initial stages (approximately $60 \%$ fractional release) [46].

$$
\frac{M_{t}}{M_{\infty}}=k t^{n}
$$

where $M_{t} / M_{\infty}$ represents the fraction of the drug released; $M_{t}$ and $M_{\infty}$ are the absolute cumulative amount of drug released at time $t$ and at infinite time, respectively (in this case the maximum amount released in the experimental conditions used, at the plateau of the release curves; $k$ is a constant incorporating the characteristics of the macromolecular drug loaded system and $n$ is the diffusional exponent characteristic for the release mechanism). 
In the equation above, a value of $n \approx 0.5$ indicates a Fickian diffusion mechanism of the drug from the hydrogel network, while a value $0.5<n<1$ indicates an anomalous or non-Fickian behavior. When $n=1$, a case II transport mechanism is involved with zero order kinetics, while $n>1$ indicates a special case II transport mechanism [47]. The corresponding SDZ release profiles are represented through plots of the cumulative percentage of drug released versus time.

\subsubsection{Antimicrobial Tests}

The antimicrobial tests were effectuated according to standard methods SR ISO 16649-2/2007 [48]. The lyophilized ATCC cultures: Salmonella typhymurium 14028, Listeria monocytogenes 7644 and Escherichia coli25922, which were purchased from American Type Culture Collection (Rockville, MD, USA) used as microorganism strains in this study. The ATCC cultures have been reconstituted according to the requirements of specific standards such as: SR EN ISO 11133/2014 [49]; ILAC G9/2005 [50]; SR EN ISO 7218-A1/2014 [51].

The lyophilized culture was subcultured to obtain replicate reference stock cultures and further the reference stock culture was subcultured to obtain the working stock culture. From this working stock culture bacterial suspensions of $0.5 \mathrm{McF}$ (measured with a densitometer) were obtained. The obtained suspensions were serial diluted to achieve concentrations of about $10^{2}-10^{3} \mathrm{UFC} / 0.1 \mathrm{~mL}$ that were used for testing the prepared polymer samples.

ATCC culture bacteria contamination, innoculation and incubation performed for $24 \mathrm{~h}$ and $48 \mathrm{~h}$ at $44{ }^{\circ} \mathrm{C}$, identifying target germs. Sterilization of the samples was made in autoclave at $110{ }^{\circ} \mathrm{C}, 0.5$ bars for $20 \mathrm{~min}$. Preparation of ATCC cultures was done by seeding the average pre-enrichment and incubation at $37^{\circ} \mathrm{C}$ for $24 \mathrm{~h}$, counting the colonies in $0.1 \mathrm{~mL}$ culture by selective culture medium separation, seeding of $0.1 \mathrm{~mL}$ bacterial culture ATCC using sterile swab samples surface.

After $24 \mathrm{~h}$ and $48 \mathrm{~h}$ using sterile tampons moistened in peptone physiological serum was collected the specimens from the test surfaces. The collected specimens were seeded on the surface of specific culture media: XLD - Xylose Lysine Desoxycholate agar for Salmonella typhymurium; ALOA-Agar Listeria Ottaviani\&Agosti for Listeria monocytogenes; and VRBG_Violet Red Bile Glucose Agar in the case of Escherichia coli and the specific colonies of each bacterial species were counted.

By knowing how much the sample was diluted prior to being plated, along with the amount of the dilution used in plating, the concentration of the viable cells per milliliter in the original sample was calculated. To be comparable, the reduction ratio of the bacteria was evaluated by the following equation: $R(\%)=\frac{A-B}{A} \times 100 \%$, where $R$ is the percentage reduction ratio; $A$ is the number of bacterial colonies from the untreated bacteria suspension (without samples to be tested); and $B$ is the number of bacterial colonies from the bacteria culture treated with samples under study.

\section{Conclusions}

Chitosan was functionalized with sulfadiazine without auxiliary molecules, enhancing its bacteriostatic effect and subsequently the ability to promote wound healing. Polyelectrolyte sponges prepared from SDZ-modified CS (SCS) with sodium hyaluronate showed an increased swelling capacity and a heterogeneous porous morphology with pores highly interconnected. In particular 
PEC Mm sample presented a significantly slower swelling rate and a more dense/compact porosity compared with other samples, correlated with a more uniform drug distribution within the matrix and the slowest sulfadiazine release rate. The samples containing CSM and especially PEC Mm allowed a more efficient entrapment of the drug within the 3D structure followed by a subsequent slower release. The prepared PECs demonstrated their antimicrobial efficiency against Escherichia coli, Listeria monocytogenes and Salmonella thyphymurium.

The results obtained demonstrated that the PECs prepared from SDZ-modified chitosan represent an attractive alternative as efficient systems for prolonged drug delivery with enhanced bacteriostatic effect.

\section{Acknowledgments}

This work was supported in part by a grant of the Romanian National Authority for Scientific Research, CNCS-UEFISCDI, project number PN-II-ID-PCE-2011-3-0906 and the grant funded by SEE 2009-2014, project number 1/23.06.2014.

\section{Author Contributions}

RPD, LP and CV designed research; RPD, OMD and LEN performed research; CV, LP and DP analyzed the data; CV, RPD and LP wrote the paper. All authors read and approved the final manuscript.

\section{Conflicts of Interest}

The authors declare no conflict of interest.

\section{References}

1. Brown, M.A.; Daya, M.R.; Worley, J.A. Experience with chitosan dressings in a civilian EMS system. J. Emerg. Med. 2009, 37, 1-7.

2. Jayakumar, R.; Prabaharan, M.; Sudheesh Kumar, P.T.; Nair, S.V.; Tamura, H. Biomaterials based on chitin and chitosan in wound dressing applications. Biotechnol. Adv. 2011, 29, 322-337.

3. Jayakumar, R.; Prabaharan, M.; Sudheesh Kumar, P.T.; Nair, S.V.; Furuike, T.; Tamura, H. Novel chitin and chitosan materials in wound dressing. In Biomedical Engineering, Trends in Materials Science; Laskovski, A.N., Ed.; InTech: Rijeka, Croatia, 2011; pp. 1-23.

4. Dai, T.; Tanaka, M.; Huang, Y.-Y.; Hamblin, M.R. Chitosan preparations for wounds and burns: Antimicrobial and wound-healing effects. Expert Rev. Anti-Infect. Ther. 2011, 9, 857-879.

5. Biagini, G.; Muzzarelli, R.A.A.; Giardino, R.; Castaldini, C. Biological materials for wound healing. In Advances in Chitin and Chitosan; Brine, C.J., Sandford, P.A., Zikakis, J.P., Eds.; Elsevier Science: Barking, UK, 1992; Volume 11, pp. 16-24.

6. Ueno, H.; Mori, T.; Fujinaga, T. Topical formulations and wound healing applications of chitosan. Adv. Drug Deliv. Rev. 2001, 52, 105-115.

7. Liu, X.F.; Guan, Y.L.; Yang, D.Z.; Li, Z.; de Yao, K. Antibacterial action of chitosan and carboxymethylated chitosan. J. Appl. Polym. Sci. 2000, 79, 1324-1335. 
8. Paul, W.; Sharma, C.P. Chitin and alginates wound dressings: A short review. Trends Biomater. Artif. Organs 2004, 18, 18-23.

9. Mi, F.-L.; Shyu, S.-S.; Wu, Y.-B.; Lee, S.-T.; Shyong, J.-Y.; Huang, R.-N. Fabrication and characterization of a sponge-like asymmetric chitosan membrane as a wound dressing. Biomaterials 2001, 22, 165-173.

10. Kim, S.J.; Shin, S.R.; Lee, S.M.; Kim, I.Y.; Kim, S.I. Thermal characteristics of polyelectrolyte complexes composed of chitosan and hyaluronic acid. J. Macromol. Sci. 2003, A40, 807-815.

11. Rinaudo, M. Properties and degradation of selected polysaccharides: Hyaluronan and CS. Corros. Eng. Sci. Technol. 2007, 42, 324-334.

12. Radaeva, I.F.; Kostina, G.A.; Il'ina, S.G.; Kostyleva, R.N. Antimicrobial activity of hyaluronic acid. Zh. Mikrobiol. Epidemiol. Immunobiol. 2001, 1, 74-75.

13. Kulkarni, A.R.; Kulkarni, V.H.; Keshavayya, J.; Hukkeri, V.I.; Sung, H.-W. Antimicrobial activity and film characterization of thiazolidinone derivatives of Chitosan. Macromol. Biosci. 2005, 5, 490-493.

14. Fang, J.Y.; Chen, J.P.; Leu, Y.L.; Hu, J.W. Temperature-sensitive hydrogels composed of CS and hyaluronic acid as injectable carriers for drug delivery. Eur. J. Pharm. Biopharm. 2008, 68, 626-636.

15. Riva, R.; Ragelle, H.; des Rieux, A.; Duhem, N.; Jerome, C.; Preat, V. Chitosan and chitosan derivatives in drug delivery and tissue engineering. Adv. Polym. Sci. 2011, 244, 19-44.

16. Prabaharan, M. Review paper: Chitosan derivatives as promising materials for controlled drug delivery. J. Biomater. Appl. 2008, 23, 5-36.

17. Bansal, V.; Sharma, P.K.; Sharma, N.; Pal O.P.; Malviya R. Applications of chitosan and chitosan derivatives in drug delivery. Adv. Biol. Res. 2011, 5, 28-37.

18. Sinha, V.R.; Kumria, R. Polysaccharides in colon-specific drug delivery. Int. J. Pharm. 2001, 224, $19-38$.

19. Onishi, H.; Shimoda, J.; Machida, Y. Chitosan-drug conjugate microspheres: Preparation and drug release properties of microspheres composed of the conjugate of 2'- or 3'-(4-Carboxy-butyryl)-5fluorouridine with chitosan. Drug Dev. Ind. Pharm. 1996, 22, 457-463.

20. Shao, X.H.; Xu, J.Q.; Jiao, Y.P.; Zhou, C.R. Synthesis and characterization of an alendronate-chitosan conjugate. Appl. Mech. Mater. 2011, 140, 53-57.

21. Yousefpour, P.; Atyabi, F.; Vasheghani-Farahani, E.; Mousavi Movahedi, A.A.; Dinarvand, R. Targeted delivery of doxorubicin-utilizing chitosan nanoparticles surface-functionalized with anti-Her2 trastuzumab. Int. J. Nanomed. 2011, 6, 1977-1990.

22. Suvannasara, P.; Juntaprama, K.; Praphairaksit, N.; Siralertmukul, K.; Muangsin, N. Mucoadhesive 4-carboxybenzenesulfonamide-chitosan with antibacterial properties. Carbohydr. Polym. 2013, 94, 244-252.

23. Muñoz-Bonilla, A.; Cerrada, M.; Fernández-García, M. Polymeric Materials with Antimicrobial Activity: From Synthesis to Applications; RSC Polymer Chemistry Series, RSC Publishing: Cambridge, UK, 2013.

24. Ong, S.Y.; Wu, J.; Moochhala, S.M.; Tan, M.H.; Lu, J. Development of a CS-based wound dressing with improved hemostatic and antimicrobial properties. Biomaterials 2008, 29, 4323-4332. 
25. Vasile, C.; Pieptu, D.; Dumitriu, R.P.; Pânzariu, A.; Profire, L. Chitosan/hyaluronic acid polyelectrolyte complex hydrogels in the management of burn wounds. Rev. Med. Chir. Soc. Med. Nat. Iasi 2013, 117, 565-571.

26. Denuziere, A.; Ferrier, D.; Damour, O.; Domard, A. Chitosan-chondroitin sulfate and chitosan-hyaluronate polyelectrolyte complexes: Biological properties. Biomaterials 1998, 19, $1275-1285$.

27. Kim, H.J.; Lee, H.C.; Oh, J.S.; Shin, B.A.; Oh, C.S.; Park, R.D.; Yang, K.S.; Cho, C.S. Polyelectrolyte complex composed of chitosan and sodium alginate for wound dressing application. J. Biomater. Sci. Polym. Ed. 1999, 10, 543-556.

28. Xu, H.; Ma, L.; Shi, H.; Gao, C.; Han, C. Chitosan-hyaluronic acid hybrid film as a novel wound dressing: In vitro and in vivo studies. Polym. Adv. Technol. 2007, 18, 869-875.

29. Rossi, S.; Marciello, M.; Sandri, G.; Ferrari, F.; Bonferoni, M.C.; Papetti, A.; Caramella, C.; Dacarro, C.; Grisoli, P. Wound dressings based on chitosans and hyaluronic acid for the release of chlorhexidine diacetate in skin ulcer therapy. Pharm. Dev. Technol. 2007, 12, 415-422.

30. Profire, L.; Pieptu, D.; Dumitriu, R.P.; Dragostin, O.; Vasile, C. Sulfadiazine modified CS/HA PEC destined to wound dressing. Rev. Med. Chir. Soc. Med. Nat. Iasi 2013, 117, 525-531.

31. Vaarum, K.M.; Smidsrod, O. Structure-property relationships in chitosans. In Polysaccharides: Structural Diversity and Functional Versatility, 2nd ed.; Dumitriu, S., Ed.; Marcel Dekker Inc.: New York, NY, USA, 2005; pp. 625-642.

32. Luppi, B.; Bigucci, F.; Mercolini, L.; Musenga, A.; Sorrenti, M.; Catenacci, L.; Zecchi, V. Novel mucoadhesive nasal inserts based on chitosan/hyaluronate polyelectrolyte complexes for peptide and protein delivery. J. Pharm. Pharmacol. 2009, 61, 151-157.

33. Burns, D.A.; Ciurczak, E.W. Handbook of Near-Infrared Analysis, 2nd ed.; Marcel Dekker Inc.: New York, NY, USA, 2001.

34. Workman, J. The Handbook of Organic Compounds: NIR, IR, Raman, and UV-VIS Spectra Featuring Polymers and Surfactans; 3-Volume Set; Academic Press: Boston, MA, USA, 2000; Volume 2, pp. 77-197.

35. Workman, J.; Weyer, L. Practical Guide to Interpretive Near-Infrared Spectroscopy, 1st ed.; CRC Press: Boca Raton, FL, USA, 2009; Volume 8, pp. 226-245.

36. Rusu-Balaita, L.; Desbrieres, J.; Rinaudo, M. Formation of a biocompatible polyelectrolyte complex: Chitosan-hyaluronan complex stability. Polym. Bull. 2003, 50, 91-98.

37. Polexe, R.C.; Delair, T. Elaboration of stable and antibody functionalized positively charged colloids by polyelectrolyte complexation between chitosan and hyaluronic acid. Molecules 2013, $18,8563-8578$.

38. Fox, C.L., Jr.; Modak, S.M.; Fox, P. Wound Dressing Comprising Silver Sulfadiazine Incorporated in Animal Tissue and Method of Preparation. U.S. Patent 4,599,226, 1977.

39. Morsi, N.; Abdelbary, G.A.; Ahmed, M.A. Silversulfadiazine based cubosome hydrogels for topical treatment of burns: Development and in vitro/in vivo characterization. Eur. J. Pharm. Biopharm. 2014, 86, 178-189.

40. Sweetman, S.C.; Blake, P.S.; McGlashan, J.M.; Parsons, A.V. Martindale: The Complete Drug Reference, 33th ed.; Pharmaceutical Press: London, UK, 2002; pp. 251-256. 
41. Dragostin, O.; Lupaşcu, F.; Vasile, C.; Baican, M.; Tuchilus, C.; Profire, L. Synthesis, characterisation and antimicrobial activity of new chitosan derivatives. In Proceeding of the BIOFUTURE 2011: Young European Biomaterial Scientists Designing: A View for the Future, Ghent, Belgium, 16-18 November 2011.

42. Feng, Y.; Xia, W. Preparation, characterization and antibacterial activity of water-soluble O-fumaryl-chitosan. Carbohydr. Polym. 2011, 83, 1169-1173.

43. Ma, G.; Yang, D.; Zhou, Y.; Xiao, M.; Kennedy, J.F.; Nie, J. Preparation and characterization of water-soluble N-alkylated chitosan. Carbohydr. Polym. 2008, 74, 121-126.

44. Batista, M.K.S.; Pinto, L.F.; Gomes, C.A.R.; Gomes, P. Novel highly-soluble peptide-chitosan polymers: Chemical synthesis and spectral characterization. Carbohydr. Polym. 2006, 64, 299-305.

45. Gupta, K.; Kumar, M.R. Preparation, characterization and release profiles of $\mathrm{pH}$-sensitive chitosan beads. Polym. Int. 2000, 49, 141-146.

46. Korsmeyer, R.W.; Lustig, S.R.; Peppas, N.A. Solute and penetrant diffusion in swellable polymers I: Mathematical modelling. J. Polym. Sci. Part B Polym. Phys. 1986, 24, 395-408.

47. Ritger, P.L.; Peppas, N.A. A simple equation for description of solute release II: Fickian and anomalous release from swellable devices. J. Control. Rel. 1987, 5, 37-42.

48. Microbiology of Food and Animal Feeding Stuff-Horizontal Method for the Enumeration of B-glucuronidase-positive Escherichia coli; Romanian Association for Standardization (ASRO): Bucharest, Romania, 2008; SR ISO 16649-2/2007.

49. Microbiology of Food, Animal Feed and Water-Preparation, Production, Storage and Performance Testing of Culture; CEN-CENELEC (European Committee for Standardization)Management Centre: Geneva, Switzerland, 2013; SR EN ISO 11133/2014.

50. Guidelines on the Selection and Use of Reference Materials; ILAC Global Trust: Sydney, Australia, 2005; ILAC G9/2005.

51. Microbiology of Food and Animal Feeding Stuffs-General Requirements and Guidance for Microbiological Examinations-Amendment 1; CEN-CENELEC (European Committee for Standardization)-Management Centre: Geneva, Switzerland, 2013; SR EN ISO 7218-A1/2014.

(C) 2015 by the authors; licensee MDPI, Basel, Switzerland. This article is an open access article distributed under the terms and conditions of the Creative Commons Attribution license (http://creativecommons.org/licenses/by/4.0/). 